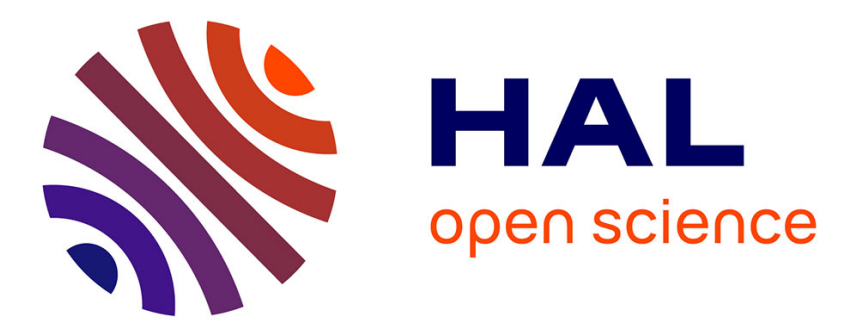

\title{
On the Use of the Hopkinson Bar for the Determination of the Transient Dynamic Behaviour of Pure Iron
}

\author{
M. Leroy, P. Louvigne, C. Nicolazo, Patrice Longère
}

\section{To cite this version:}

M. Leroy, P. Louvigne, C. Nicolazo, Patrice Longère. On the Use of the Hopkinson Bar for the Determination of the Transient Dynamic Behaviour of Pure Iron. EURODYMAT 1997 - 5th International Conference on Mechanical and Physical Behaviour of Materials under Dynamic Loading, Aug 1997, Toledo, Spain. pp.C3-259-C3-264, 10.1051/jp4:1997346 . jpa-00255503

\section{HAL Id: jpa-00255503 https://hal.science/jpa-00255503}

Submitted on 1 Jan 1997

HAL is a multi-disciplinary open access archive for the deposit and dissemination of scientific research documents, whether they are published or not. The documents may come from teaching and research institutions in France or abroad, or from public or private research centers.
L'archive ouverte pluridisciplinaire HAL, est destinée au dépôt et à la diffusion de documents scientifiques de niveau recherche, publiés ou non, émanant des établissements d'enseignement et de recherche français ou étrangers, des laboratoires publics ou privés. 


\title{
On the Use of the Hopkinson Bar for the Determination of the Transient Dynamic Behaviour of Pure Iron
}

\author{
M. Leroy, P.F. Louvigne*, C. Nicolazo and P. Longere \\ Institut Univestitaire de Technologie de Nantes, 3 rue du Maréchal Joffre, BP. 34103, \\ 44041 Nantes cedex 1, France \\ *DGA/DRET/ETCA, Centre de Recherches et d'Études d'Arcueil, 16 bis avenue Prieur de la Côte d'Or, \\ 94114 Arcueil, France
}

\begin{abstract}
The split Hopkinson pressure bar device is used to study the dynamic response of pure cold-drawn end annealing irons to a sudden decrease of strain ratc. Different strain rate history effects are pointed up for the both cold-drawn cnd annealing states. Applying different unloads to several dynamic microstructural states, allows to access the internal stresses and to characterize the main deformation mechanisms. These mechanisms are firstly thermally- activated then they change into viscous-drag mechanisms when the internal stress saturates and the strain rate become high enough.
\end{abstract}

\begin{abstract}
Résumé. Le montage de compression par barres d'Hopkinson est utilisé pour étudier la réponse dynamique du fer pur écroui et recuit à une brusque variation de vitesse de déformation. Des effets différents de l'histoire de la vitesse de déformation. pour les états écrouis et recuits, sont mis en évidences. Différents déchargements appliqués à plusieurs états microstructuraux dynamiques permettent d'ćvaluer les contraintes internes et de montrer que les mécanismes de déformation prédominants, d'abord activés thermiquement deviennent des mécanismes de frottement visqueux lorsque la contrainte interne est forte et que la vitesse de déformation est suffisamment élevée.
\end{abstract}

\section{INTRODUCTION}

De nombreuses études utilisant le principe du saut de vitesse de déformation au moyen des barres d'Hopkinson ont été menées pour montrer l'effet de l'histoire de la déformation sur le comportement du matériau et relier ces observations macroscopiques à l'évolution de la microstructure. Certains travaux sont basés sur le chargement statique-dynamique en torsion (Campbell et Dowling [1], Nicholas [2], Frantz et Duffy [3], Senseny et al. [4], Harding [5] et Klepaczko [6]) consistant en l'application d'un chargement quasi-statique auquel on superpose brutalement un chargement dynamique et d'autres analysent le déchargement dynamique-dynamique en torsion (Lipkin et al. [7] et Gilat et Pao [8]).

L'objectif de cette étude est l'analyse du comportement dynamique d'un matériau, ici en l'occurrence le fer Armco à des déchargements variables en intensités effectués au cours de sa déformation dynamique. Les réponses aux divers déchargements imposés permettent d'obtenir des renseignements sur l'état microstructural dynamique de la matière en écoulement. Dans cet article, suite aux études de Leroy et al.[9], le saut dynamique-dynamique en compression est utilisé en provoquant des sollicitations par l'action de projectiles étagés permettant les déchargements souhaités. Ces sauts dynamique-dynamique sont réalisés sur un montage en barres d'Hopkinson.

Nous analysons l'effet de l'histoire de la déformation pour deux états microstructuraux à même taille de grains avant chocs : le fer pur polycristallin (cc) à l'état écroui et ou recuit. Plusieurs déchargements d'intensités variables sont appliqués à un même état microstructural dynamique. L'effèt de l'histoire de la déformation de ces états est montré et, l'étude des réponses permet d'évaluer les paramètres contrôlant son évolution.

Une comparaison est effectuée entre divers résultats de travaux [10] concernant le comportement en chargements directs et ceux obtenus par sauts dynamique-dynamique. En effet, par cette technique, les réponses aux sauts fournissent des renseignements sur les contraintes de résistance interne de la structure en évolution dynamique ainsi que sur l'apport des contraintes effectives aux déformations et aux mécanismes contrôlant celles-ci. 


\section{EXPERIMENTATION}

Le montage des barres d'Hopkinson en compression est utilisé pour ces expériences. Le déchargement dynamique-dynamique est obtenu avec un projectile étagé (figure 1).

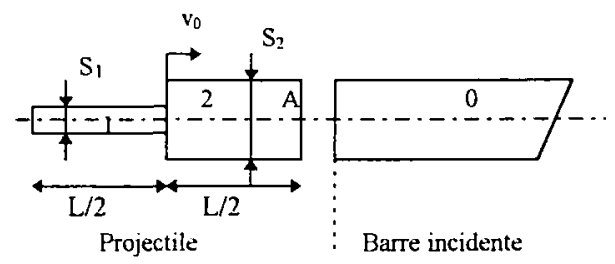

Figure 1 : Géométrie du projectile

L'impact produit une contrainte incidente qui atteint son maximum en environ $12 \mu \mathrm{s}$. Durant le chargement correspondant à l'impulsion $\mathrm{J}_{1}$, le métal est déformé à une vitesse de déformation plastique $\dot{\varepsilon}_{1}$ jusqu'à un taux de déformation plastique $\varepsilon_{\mathrm{s}}$. Pendant cette déformation dynamique le métal est brusquement décharge avec une seconde impulsion $\mathrm{J}_{2}<\mathrm{J}_{\mathrm{l}}$. La réponse du matériau à ce déchargement est un saut de vitesse de déformation et un saut de contrainte liés à l'état microstructural dynamique à l'instant du saut $\left(\dot{\varepsilon}_{1}, \varepsilon_{s}, \sigma, \mathrm{T}\right)$. Pour caractériser un état microstructural, on effectue une série de déchargements avec plusieurs projectiles de même longueur et de section $S_{1}$ différentes. On obtient la même impulsion $J_{1}$ donc la même vitesse $\dot{\varepsilon}_{1}$ et le même taux de déformation $\varepsilon_{\mathrm{s}}$, suivie d'une impulsion $J_{2}$ de même durée mais d'intensité différente d'où une vitesse $\dot{\varepsilon}_{2}$ différente (figures 2 et 3 ).

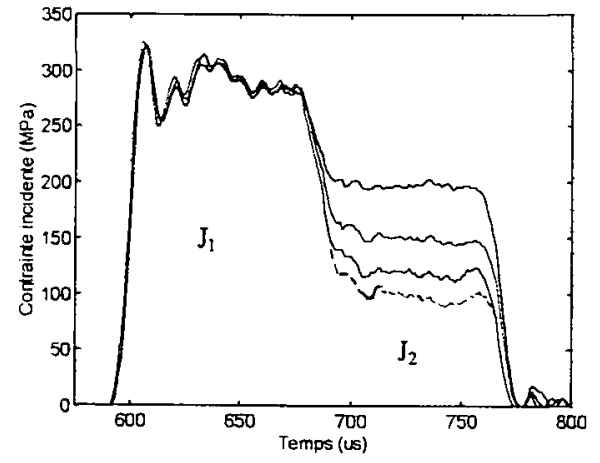

Figure 2 : Onde de contrainte incidente : impulsions $\mathrm{J}_{1}$ et $\mathrm{J}_{2}$.

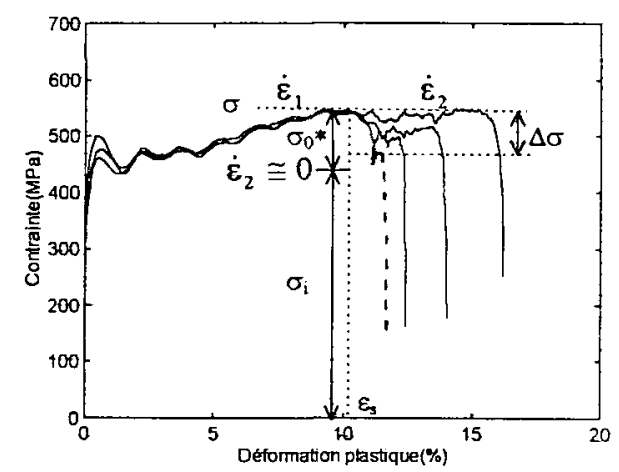

Figure 3 : Comportement du Fer recuit en déchargement

Le matériau étudié est le fer polycristallin à l'état écroui et à l'état recuit. Le fer est fourni à l'état écroui. de dureté Vickers voisine de $200 \mathrm{HV}$ et de taille de grain d'environ $17 \mu \mathrm{m}$. Le but du recuit est d'éliminer les défauts sans modifier la taille du grain. Ceci est obtenu après un recuit de 2 heures à $500^{\circ} \mathrm{C}$, la durete Vickers devenant voisine de $90 \mathrm{HV}$. Les échantillons sont des cylindres pleins de $8 \mathrm{~mm}$ de diamètre par 8 $\mathrm{mm}$ de longueur.

\section{RESULTATS ET DISCUSSION}

\subsection{Contrainte interne ou athermique}

Au cours de la déformation dynamique, le matériau a une résistance due aux contraintes internes. Ces contraintes internes sont liées aux diverses actions de la microstructure sur les dislocations. Lors de la déformation plastique, la contrainte $\sigma$ est la somme de la contrainte interne qu'il faut vainere pour commencer à le plastifier, et de la contrainte efficace $\sigma^{*}$ qui amène le matériau à se déformer à une vitesse 
$\dot{\varepsilon}$. La figure 3 illustre des sauts dynamique-dynamique, sauts permettant d'évaluer la contrainte interne ou athermique $\sigma_{i}$ par application d'impulsions $J_{2}$ d'intensités décroissantes jusqu'à $\dot{\varepsilon}_{2} \cong 0$. L'extrapolation selon $\dot{\varepsilon}_{2}$ des variations de $\Delta \sigma$ aux sauts pour $\dot{\varepsilon}_{2} \rightarrow 0$ permet de déterminer la contrainte efficace $\sigma_{0}{ }^{*}$, valeurs de $\sigma_{\mathrm{i}}$ et $\sigma_{0}{ }^{*}$ spécifiques de l'état structural $\left(\dot{\varepsilon}_{1}, \varepsilon_{\mathrm{s}}, \sigma, \mathrm{T}\right)$.

Remarque : un des modèles [10] introduit dans les codes de calcul pour résoudre les problèmes d'impact est par exemple :

$$
\sigma=\Delta \sigma_{G}{ }^{\prime}+C_{1} \exp \left(-C_{3} T+C_{4} T \ln \dot{\varepsilon}\right)+C_{5} \varepsilon^{a}+C_{6} 1^{-1 / 2}
$$

avec pour les cfc : $C_{1}=C_{2} \varepsilon^{1 / 2}$ et $C_{5}=0$ et pour les cc : $C_{1}$ indépendant de $\varepsilon$, pour le fer Armco $\Delta \sigma_{G}^{\prime} \cong 0$ La relation (1) est séparable en deux composantes : une composante athermique $\sigma_{\text {ath }}$ que l'on pourra comparer à la contrainte interne $\sigma_{\text {; }}$ et une composante thermique $\sigma_{\mathrm{th}}$ comparée à la contrainte efficace $\sigma^{*}$.

$$
\begin{aligned}
& \sigma_{a \mathrm{ath}}=\Delta \sigma_{\mathrm{G}}{ }^{1}+\mathrm{C}_{5} \varepsilon^{\mathrm{n}}+\mathrm{C}_{6}{ }^{1 / 2} \\
& \sigma_{\mathrm{th}}=\mathrm{C}_{1} \exp \left(-\mathrm{C}_{3} \mathrm{~T}+\mathrm{C}_{4} \mathrm{~T} \ln \dot{\varepsilon}\right)
\end{aligned}
$$

Lorsque l'on calcule $\sigma_{\text {ath }}$ en prenant $\Delta \sigma_{\mathrm{G}^{\prime}}, \mathrm{C}_{5}$, et $\mathrm{C}_{6}$ déterminés par les auteurs [10] pour le fer Armco, et nos valeurs de $n$ et $l$, nous obtenons $\sigma_{\text {th }}<\sigma_{\mathrm{i}}$ expérimental avec des variations respectivement de 30 à $40 \%$ pour le Fe recuit et le Fe écroui. Les valeurs de $\Delta \sigma_{G}{ }^{\prime}, C_{5}$, et $C_{6}$ sont supposées constantes durant l'évolution

\begin{tabular}{|c|c|c|c|c|c|c|}
\hline & $\dot{\varepsilon}_{1}\left(s^{-1}\right)$ et $\varepsilon_{s}(\%)$ & $\sigma(\mathrm{MPa})$ & $\sigma_{0} *(\mathrm{MPa})$ & $\sigma_{1}(\mathrm{MPa})$ & $\frac{\mathrm{V}}{\mathrm{kT}}$ en $\mathrm{MPa}^{-1}$ & $V$ en $10^{-9} \mu \mathrm{m}^{3}$ \\
\hline \multirow{5}{*}{ Ecroui } & $2500-17$ & 800 & 140 & 660 & 0.037 & 0.167 \\
\hline & $2500-14$ & 800 & 140 & 660 & 0.037 & 0.165 \\
\hline & $2500-10$ & 800 & 140 & 660 & 0.037 & 0.160 \\
\hline & $1890-14$ & 770 & 110 & 660 & 0.047 & 0.208 \\
\hline & $1600-10$ & 730 & 110 & 620 & 0.047 & 0.201 \\
\hline \multirow{5}{*}{ Recuit } & $2500-15$ & 606 & 140 & 466 & 0.024 & 0.104 \\
\hline & $2500-10$ & 580 & 140 & 440 & 0.024 & 0.102 \\
\hline & $1600-10$ & 542 & 110 & 432 & 0.038 & 0.160 \\
\hline & $.1400-10$ & 545 & 110 & 435 & 0.038 & 0.160 \\
\hline & $1220-10$ & 538 & 110 & 428 & 0.038 & 0.160 \\
\hline
\end{tabular}
dynamique du matériau ce qui, à priori, ne semble pas être le cas pour nos valeurs de $\sigma_{i}$ (tableau 1).

Tablcau 1: Récapitulatif des résultats

\subsection{Contrainte efficace ou thermique}

\subsubsection{Mise en évidence du mécanisme de déformation}

Le saut dynamique-dynamique permettrait de mettre en évidence certains paramètres contrôlant la déformation. En effet, lorsque la vitesse de déformation $\dot{\varepsilon}$ est due uniquement à un mécanisme thermiquement activé, la relation fondamentale employée est :

$$
\dot{\varepsilon}=\dot{\varepsilon}_{0} \exp \left(-\frac{\Delta \mathrm{G}^{*}}{\mathrm{kT}}\right) \quad \text { avec } \Delta \mathrm{G}^{*}=\Delta \mathrm{G}-\sigma^{*} \mathrm{~V}
$$

où $\dot{\varepsilon}_{0}$ est un paramètre proportionnel à la densité de dislocations mobiles, $\mathrm{T}$ la température, $\mathrm{k}$ la constante de Boltzmann, $\Delta G^{*}$ l'énergie d'activation, $\Delta G$ l'énergie moyenne des obstacles à franchir et $V$ le volume d'activation. 
De même, pour des vitesses de déformation plus élevées, le mécanisme principal contrôlant la déformation est supposé dû à la viscosité $\eta(T)$ du milieu. Sachant que le terme d'amortissement visqueux B [11-12] est relié à la vitesse moyenne $\overline{\mathrm{v}}$ des dislocations mobiles en nombre $\mathrm{N}$ nous avons :

$$
\mathrm{b} \tau_{\mathrm{B}}{ }^{*}=\mathrm{b} \frac{\sigma_{\mathrm{B}}^{*}}{\mathrm{~m}}=\mathrm{B}_{(\mathrm{r})} \bar{v} \text { et } \dot{\gamma}=\mathrm{m} \dot{\varepsilon}=\mathrm{Nb} \bar{v} \text { d'où } \sigma_{\mathrm{B}}^{*}=\left(\frac{\mathrm{m}}{\mathrm{b}}\right)^{2} \frac{\mathrm{B}}{\mathrm{T}(\mathrm{T})} \dot{\varepsilon}_{\mathrm{B}}=\mathrm{C}_{7} \frac{\mathrm{B}_{(\mathrm{T})}}{\mathrm{N}} \dot{\varepsilon}_{\mathrm{B}}
$$

Dans ce cas la relation employée aux sauts aux grandes vitesses serait de la forme :

$$
\dot{\varepsilon}_{\mathrm{B}}=\mathrm{C} \sigma_{\mathrm{B}}^{*}
$$

Afin de mettre en évidence ces mécanismes, nous traçons $\Delta \ln \dot{\varepsilon}$ aux sauts en fonction de $\Delta \sigma$ pour chaque état microstructural dynamique étudié et à $\dot{\varepsilon}_{1}$ fixées.

Valeurs expérimentales de V/kT obtenues par des sauts en déchargements dynamique-dynamique à diverses vitesses el taux de déformations plastiques.

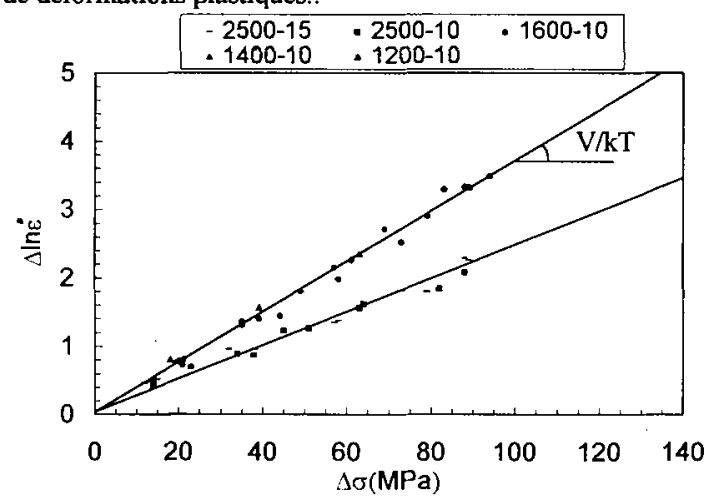

Figure 4a: Cas du Fe recuit

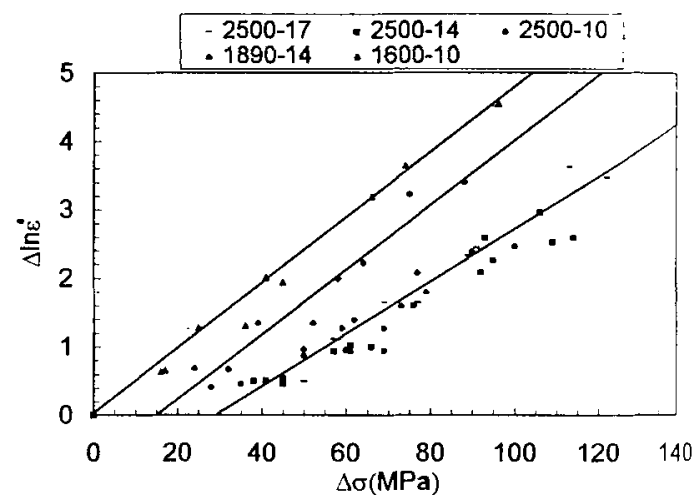

Figure 4b: Cas du Fe ćcroui

Les courbes figures $4 a$ et $4 b$ montrent pour un grand nombre d'essais $(>100)$ et, malgré quelques dispersions expérimentales, que nous pouvons tracer des droites pour toutes les réponses aux sauts affectées à chaque état microstructural dynamique.

Pour le Fe recuit et écroui à $\dot{\varepsilon}_{1}<1600 \mathrm{~s}^{-1}$, toutes les droites passent par l'origine. Le mécanisme de déformation serait donc thermiquement activé avant et après les sauts. En effet, si on considère que $\dot{\varepsilon}_{0}$ et $T$ restent constants aux déchargements :

$$
\Delta \ln \dot{\varepsilon}=\ln \dot{\varepsilon}_{1}-\ln \dot{\varepsilon}_{2}=\frac{\mathrm{V}}{\mathrm{kT}} \Delta \sigma^{*}=\frac{\mathrm{V}}{\mathrm{kT}} \Delta \sigma
$$

en notant que $\Delta \sigma^{*}=\sigma_{0}^{*}-\sigma^{*}=\Delta \sigma$

Ce fait est aussi constaté pour des essais sur le fer recuit jusqu'à $2500 \mathrm{~s}^{-1}$.

La pente des droites nous donne les rapports V/kT (tableau 1).

Pour Fe écroui avec $\dot{\varepsilon}_{1} \geq 1900 \mathrm{~s}^{-1}$ les droites ne passent pas par l'origine, ce qui laisse supposer qu'avant le saut, le mécanisme de déformation prédominant est dû au frottement visqueux (5) d'où :

$$
\Delta \ln \dot{\varepsilon}=\ln \dot{\varepsilon}_{1}-\ln \dot{\varepsilon}_{2}=\ln C \sigma_{\mathrm{B}}^{*}-\ln \left(\dot{\varepsilon}_{0} \exp \left(-\frac{\Delta \mathrm{G}-\mathrm{V} \sigma_{0}^{*}}{\mathrm{kT}}\right)\right)+\frac{\mathrm{V}}{\mathrm{kT}} \Delta \sigma^{*}(7)
$$

avec $\ln C \sigma_{\mathrm{B}}^{*}-\ln \left(\dot{\varepsilon}_{0} \exp \left(-\frac{\Delta \mathrm{G}-\mathrm{V} \sigma_{0}^{*}}{\mathrm{kT}}\right)\right)$ indépendant de $\Delta \sigma^{*}$.

La pente des droites nous donne aussi le rapport V/kT pour des dechargements aux plus faibles vitesses 


\subsubsection{Volume d'activation}

En estimant que $90 \%$ du travail plastique est transformé en chaleur [13], on évalue la température au saut ainsi que le volume d'activation $\mathrm{V}$ (tableau 1). Nous constatons de faibles variations d'échauffement $\left(10^{\circ}<\Delta \mathrm{T}<40^{\circ}\right)$ et dans ce cas, les volumes d'activation déduits sont compris entre 0.1 et $0.2 .10^{-9} \mu \mathrm{m}^{3}$ (tableau 1) avec pour $\dot{\varepsilon}_{1}$ augmentant, des valeurs de V diminuant dans des rapports de l'ordre de 1.6 et 1.2 pour Fe recuit et Fe écroui.

\subsubsection{Comparaison de $\sigma_{\text {th }}$ et $\sigma^{*}$ et mise en évidence de l'effet de l'histoire de la déformation}

En appliquant une série de sauts à un mème état microstrucural ( $\dot{\varepsilon}_{1}$ reste fixe), la relation (4) nous donne :

$$
\Delta \sigma^{*}=\Delta \sigma=\frac{k T}{V}\left(\ln \dot{\varepsilon}_{1}-\ln \dot{\varepsilon}_{2}\right)
$$

Pour les mêmes sauts de vitesses de déformation nous allons comparer cette variation de contrainte efficace expérimentale à celle de contrainte thermique donnée par la relation (3) :

$$
\Delta \sigma_{\mathrm{th}}=\mathrm{C}_{1} \exp \left(-\mathrm{C}_{5} \mathrm{~T}\right)\left[\exp \left(\mathrm{C}_{4} \mathrm{~T} \ln \dot{\varepsilon}_{1}\right)-\exp \left(\mathrm{C}_{4} \mathrm{~T} \ln \dot{\varepsilon}_{2}\right)\right]
$$

Nous traçons pour cela les variations expérimentales $\Delta \sigma=\mathrm{f}\left(\ln \dot{\varepsilon}_{2}\right)$ et $\Delta \sigma_{t h}=\mathrm{f}\left(\ln \dot{\varepsilon}_{2}\right)$ donnée par (9).

Exemples d’études comparatives entre les valcurs cxpérimentales (traits pleins) aux sauts dỵnamique-dynamique de $\Delta \sigma^{*}$ et les valeurs $\Delta \sigma_{\text {th }}$ calculées (traits mixtes) déduites des travaux de Zerilli et Armstrong [10]

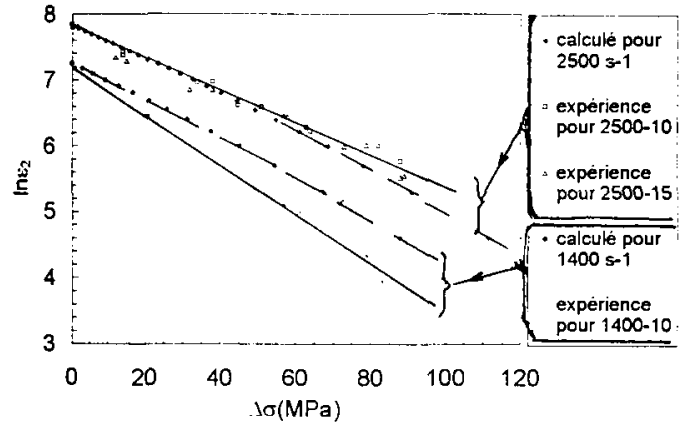

Figure 5a : cas du Fe recuit à $\dot{\varepsilon}_{1}$ valant 1400 et $2500 \mathrm{~s}^{-1}$

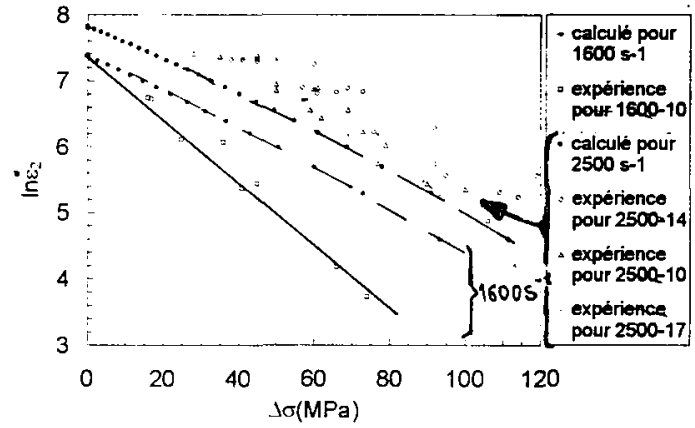

Figure 5b : cas du Fe écroui à $\dot{\varepsilon}_{1}$ valant 1600 et $2500 \mathrm{~s}^{-1}$ (avec pour $2500 \mathrm{~s}^{-1} \mathrm{l}^{*}$ hypothèse sans frottement visqueux)

Les figures $5 \mathrm{a}$ et $5 \mathrm{~b}$ montrent que les courbes divergent : plus le saut de vitesse est important plus l'écart entre les variations de contraintes $\Delta \sigma_{t_{h}}$ et $\Delta \sigma^{*}$ est grand.

Remarques : ces écarts entre les courbes valorisent le fait que les variations $\Delta \sigma^{*}$ sont dues à de mêmes états structuraux aux sauts tandis que les $\Delta \sigma_{\text {th }}$ correspondent à des comparatifs de sauts de vitesses sans tenir compte d'une évolution structurale possible entre divers chargements directs.

Cette différence renforcerait la mise en évidence de l'effet de l'histoire de la déformation. De même, un autre constat intéressant est la comparaison parmi tous les essais de ceux relatifs à deux échantillons de même structure initiale, déformés à même taux et ayant des vitesses de déchargements $\dot{\varepsilon}_{2}$ identiques. L'effet de l'histoire de la déformation serait ainsi mis en évidence par des valeurs différentes de contraintes après le saut à même $\dot{\varepsilon}_{2}$. Deux exemples figures $6 \mathrm{a}$ et $6 \mathrm{~b}$ illustrent notre hypothèse, en effet, les niveaux sont différents avec des effets inverses pour l'état recuit et l'état écroui : le niveau le plus bas avant le saut devient le plus élevé après le saut pour l'état recuit alors que le niveau le plus bas reste le plus bas après le saut pour l'état écroui. 


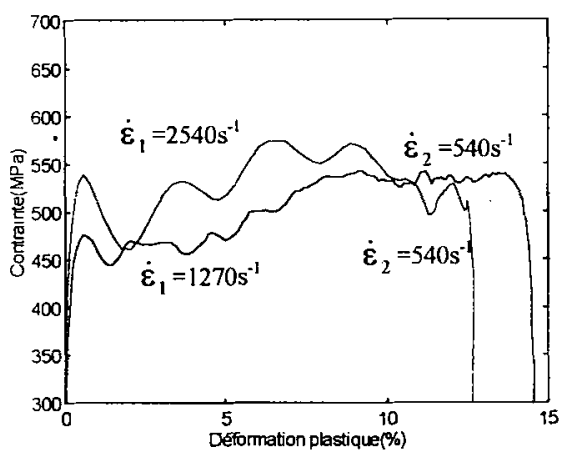

Figure 6a: Cas du Fe recuit

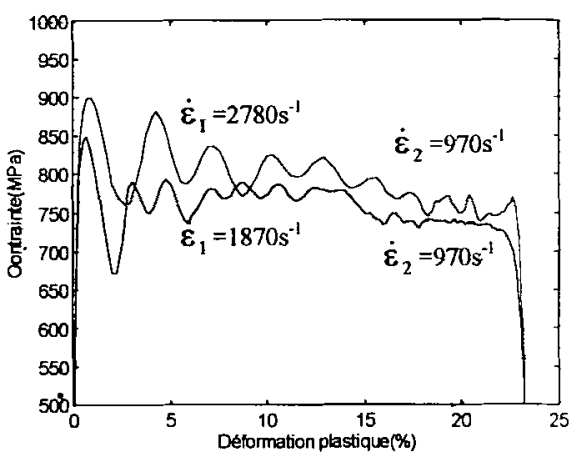

Figure 6b : Cas du Fe ćcroui

\section{CONCLUSION}

La prise en compte de la mémoire structurale des états dynamiques présente un intérêt pour la compréhension des mécanismes contrôlant la déformation et l'établissement des lois de comportement (détermination expérimentale des paramètres de ces lois). Les résultats obtenus par les essais de sauts dynamique-dynamique sur le fer à l'état recuit et ou écroui ont permis de connaître les niveaux des contraintes internes de la structure ainsi que les volumes d'activation jusqu'à $2500 \mathrm{~s}^{-1}$.

De manière générale, nous constatons des différences entre nos valeurs en déchargements et celles obtenues par d'autres auteurs avec, notamment, des écarts pour le Fer écroui, à forte contrainte interne, écarts aux grandes vitesses sans doute dû au frottement visqueux.

\section{Remerciements.}

Les auteurs remercient J.C. Pascual (IUT, Université de Nantes) et $J$. Clisșon (DGA/DCE/CREA, Paris) pour leur soutien technique.

\section{Références}

[1] Campbell J.D., Dowling A.R., J. Mech.Phys. Solids, 18 (1970) 43-63.

[2] Nicholas T., Experimental mechanics, $11(8)$ (1971) 370-374.

[3] Frantz R.A., Duffy J., ASME J. Appl. Mech., 39 (1972) 939-945.

[4] Senseny P.E., Duffy J., Hawley R.H. ., ASME J. Appl. Mech., 45 (1978) 60-66.

[5] Harding J., Mechanical Properties at High Rates of Strain (Ed. J. Harding, The Inst. of Phys., London, 1974b) pp 190-191.

[6] Klepaczko J., Mat. Sc. Eng., 18 (1975) 121-135.

[7] Lipkin J., Campbell J.D., Swearengen J.D., J. Mech. Phys. Solids, 26 (1978) 251-268.

[8] Gilat A., Pao Y.H., Exp. Mech., 28 (1988) 322-325.

[9] Leroy M., Raad M.K., Nkule L., Cheron R., Mechanical Properties at High Rates of Strain (Ed. J. Harding, The Inst. of Phys., Bristol and London, 1984) pp 31-38.

[10] Zerilli F.J., Armstrong R.W., J. Appl. Phys., 61(5) (1987) 1816-1825.

[11] Leroy M., Offret S., C. R. Acad. Sc. Paris, Série C,(27 oct. 1971).

[12] Leroy M., Thèse, Ref. CNRS AO-76 59, ENSM Univ. Nantes,(1972).

[13] Taylor et Quinney, proceeding of the Royal Society of London, A413 (1934), page 307 à 326 